\title{
Comparison of acute phase protein and hemodynamic variables in dogs undergoing video-assisted thoracoscopic vs. open pneumonectomy
}

\author{
HAI-FENG LIU, QING-MING REN, ZHI-BO WANG, XIN LI, \\ SHENG JIANG, JIAN-TAO ZHANG and HONG-BIN WANG \\ Department of Veterinary Surgery, College of Veterinary Medicine, \\ Northeast Agricultural University, Harbin, Heilongjiang 150030, P.R. China
}

Received August 10, 2015; Accepted January 20, 2017

DOI: $10.3892 /$ etm.2017.4257

\begin{abstract}
It has been demonstrated that video-assisted thoracoscopic surgery (VATS) is feasible and safe in humans and animal models. The aim of the present study was to compare the surgical outcome using VATS with that of the standard transthoracic approach for pneumonectomy in dogs, to determine the acute-phase reaction in VATS pneumonectomy, and to analyze the difference between VATS and the standard transthoracic approach. A total of 14 dogs were divided into two groups $(n=7)$; one group underwent VATS and the other group underwent a transthoracic pneumonectomy. Pre-, intraand post-operative physiologic parameters were monitored, in addition to the blood cell count and serum acute-phase protein (APP) concentrations. The APP and hemodynamic changes between the two approaches were analyzed. Mean surgical time in the VATS group (176.7 min) was significantly longer compared with the open group (132.4 min). All APP concentrations were significantly increased at day 1 postoperation and gradually decreased to preoperative concentrations. The serum concentration of C-reactive protein on day 3 and the white blood cell count on day 1 were significantly higher following surgery in the open group compared with the VATS group $(\mathrm{P}<0.05)$. No differences were observed in the physiological parameters between the two groups. Although VATS took longer, animals experienced smaller incision and less stress. Therefore, the VATS approach was satisfactory for total pneumonectomy.
\end{abstract}

Correspondence to: Dr Hong-Bin Wang, Department of Veterinary Surgery, College of Veterinary Medicine, Northeast Agricultural University, 59 Mucai Street, Gongbin Road, Xiangfang, Harbin, Heilongjiang 150030, P.R. China

E-mail: hbwang1940@163.com

Key words: video-assisted thoracoscopic surgery, pneumonectomy, acute phase protein, hemodynamic, thoracic cavity

\section{Introduction}

Video-assisted thoracoscopic surgery (VATS) has been used as a diagnostic and therapeutic platform to perform a wide variety of thoracic cavity surgical procedures (1), including surgical lung biopsy, cancer staging, pneumothorax, pericardiotomy and pericardial window creation (2). VATS is clinically accepted as being less painful and causing less stress than traditional open procedures. Eliminating conventional abdominal incisions is considered to have a number of potential advantages including faster recovery and thus a shorter hospital stay, decreased postoperative pain, improved postoperative pulmonary function and improved cosmetic outcome. Previous studies have also confirmed the safety and benefits of VATS in thoracic cavity surgery $(3,4)$.

Peumonectomy is a lung resection technique used in humans and animals to remove all lung lobes when bilobectomy or lobectomy techniques are inadequate to remove the pathology in the hemithorax. Pneumonectomy was performed to treat certain pathological conditions including lung tumors, congenital lung anomalies, chronic lung collapse, chronic progressive lung inflammation, post-traumatic diffuse parenchymal laceration and bronchial rupture $(5,6)$.

A number of comparative studies on VATS procedures in animals have evaluated stress parameters and postoperative outcomes $(7,8)$; there have also been studies into the outcomes of VATS lobectomy $(4,9)$. Compared with VATS, hemithorax pneumonectomy is more difficult to perform and there is a higher risk of intra- and post-operative complications occurring (10); thus, the degree of stress and the postoperative hemodynamics in VATS compared with traditional open pneumonectomy remains to be evaluated. Study.

Acute-phase proteins (APPs) are a series of proteins that are sensitive to inflammation and body stress including infection, surgical trauma, certain diseases and tissue damage (11). The concentrations of certain APPs, including C-reactive protein (CRP) and serum amyloid A (SAA), may increase markedly during stress or under pathological conditions. YKL-40 is another type of APP that increases in proportion to body stress, indicating the respiratory function level and pneumopathy prognosis. 
To thoroughly assess the impact of minimally invasive surgical techniques on acute-phase reactions, metabolic changes and stress responses, the current study compared such factors in dogs undergoing pneumonectomy via the VATS approach vs. those undergoing open thoracotomy to compare the impacts of the two approaches.

\section{Materials and methods}

Animals. A total of 14 mongrel dogs aged 2-5 years with a mean body weight of $7.7 \mathrm{~kg}$ (range $5.4-10.5 \mathrm{~kg}$ ) were used in the present study. The dogs were obtained from the Experimental Animal Center of Northeast Agricultural University (Harbin, China). The dogs were housed at a controlled temperature $\left(20^{\circ} \mathrm{C}\right)$ and humidity $\left(60^{\circ} \mathrm{C}\right)$ under a $12 \mathrm{~h} \mathrm{light/dark} \mathrm{cycle.} \mathrm{All}$ animals were fed a standard canine diet 3 times daily and had free access to water. Of the 14 dogs enrolled in the study, 9 were ultimately adopted and 5 were euthanized. Dogs were sacrificed via $300 \mathrm{mg}$ intravenous xylocaine (Shandong Jincheng Pharmaceutical Co., Ltd., Zibo, China) following anesthetizing with $0.1 \mathrm{mg} / \mathrm{kg}$ xylazine and $5 \mathrm{mg} / \mathrm{kg}$ ketamine hydrochloride (Jiangsu Hengrui Medicine Co., Ltd., Lianyungang, China). A preoperative physical examination and complete blood count indicated that all dogs were healthy. Animals were randomly and equally divided into two groups $(n=7)$ : One group underwent a VATS pneumonectomy and the other group underwent an open pneumonectomy. The present study was approved by the Northeast Agricultural University Institutional Animal Care and Use Committee (Harbin, China), and was conducted in a manner consistent with the U.S. National Institutes of Health 'Guide for the Care and Use of Laboratory Animals', the Animal Welfare Acts (12) and the Guide for the Care and Use of Agricultural Animals in Agricultural Research and Teaching, including appropriate methods of euthanasia (13).

Surgical preparation. Baseline physiological parameters were determined prior to surgery $(\mathrm{t}=0)$, including routine blood testing, heart rate (HR), respiratory frequency (RF), blood pressure $(\mathrm{BP})$, rectal temperature and serum concentrations of CRP, SAA and YKL-40. Following a $24 \mathrm{~h}$ fast and a $2 \mathrm{~h}$ water-fast, all animals were administered the same general anesthesia and were monitored and managed similarly.

Cefazolin (20 mg/kg; Harbin Pharmaceutical Group Co., Ltd., General Pharm Factory, Harbin, China) was administered intramuscularly (IM) $30 \mathrm{~min}$ prior to surgery to prevent postoperative infection. Each dog was pre-medicated with $0.05 \mathrm{mg} / \mathrm{kg}$ atropine (Beijing Shuanghe Pharmaceutical Co., Ltd., Beijing, China) subcutaneously and $\sim 15 \mathrm{~min}$ later anesthesia was induced with intravenous propofol (4-5 mg/kg; Xi'an Libang Pharmaceutical Co., Ltd., Xian, China) and midazolam (0.05-0.06 mg/kg; Jiangsu Shenhua Pharmaceutical Co., Ltd., Jiangsu, China). The dog was then placed in the supine position and intubated with a tracheal cannula. General anesthesia was maintained with $1.5-2 \%$ isoflurane (Hebei Welcome Pharmaceutical Co., Ltd., Shijianzhuang, China) gas mixed in oxygen, and respiration was maintained via mechanical ventilation with the anesthetic gas machine (respiratory frequency 12 breaths per minute, tidal volume $15-20 \mathrm{ml} / \mathrm{kg}$, inspiratory:expiratory ratio $1: 2$ ). Tramadol (4 mg/kg; Zhejiang Jiuxu Pharmaceutical Co., Ltd.,
Hangzhou, China) was administrated intravenously prior to surgery for analgesia.

The surgical site (left chest from the clavicle to the last rib and from the sternum to the spine) was shaved, aseptically prepared and draped for surgery. A heating blanket was placed between the animal and the operating table.

Sterile instruments were used for all open and laparoscopic procedures. For the VATS procedures, the endoscopes and other equipment underwent a high-level of disinfection.

VATS procedure. During the VATS procedure, 2 portals were created: The endoscope portal (portal 1) and the surgical portal (portal 2). Portal 1 (1 cm diameter) was created by a trocar inside a metal tube, and was located between the eighth and ninth rib. Portal 2 (2-3 cm diameter) was created using a scalpel and endotherm knife, and was located between the fifth and the sixth rib. A 10/11 mm trocar-cannula unit (Hangzhou Optcla Medical Instrument Co., Ltd., Hangzhou, China) was inserted through the chest wall at portal 1 . An endoscope $\left(0^{\circ}\right.$, $10 \mathrm{~mm}$ diameter, $330 \mathrm{~mm}$ long; Olympus Corporation, Tokyo, Japan) attached to a video endoscopic camera and light source (Olympus Corporation) was then advanced through this tube into the chest cavity. The margin of portal 2 was treated with an electric coagulation knife for hemostasis.

Lung-grasping forceps were used to lift the lung lobe and expose the hilum of the lung. The pulmonary artery, pulmonary vein and bronchus were separated cautiously using hemostatic forceps and peanut sponges (a homemade tool used for dissociated soft tissue). The pulmonary artery and pulmonary veins were clipped and cut off, and the bronchus was cut off following double ligation. Bronchus ligation required an extra transfixion ligation. All lung lobes in the left chest were excised in the following order: Middle lobe, upper lobe and subjacent lobe. Warm physiological saline (100-200 $\mathrm{ml}$ at $\sim 40^{\circ} \mathrm{C}$ ) was used to check the bronchus ligation and syringe the thoracic cavity; this solution was then extracted using a vacuum absorber.

Open procedure. A 7-8 cm incision was created using a scalpel and electric coagulation knife between the seventh and eighth rib. An endotherm knife was used for blood coagulation. The surgical room was expanded through a wound spreader. The surgical procedure and the order that lung lobes were excised was the same as for the VATS group. Lung-grasping forceps were used to lift the lung lobe and expose the hilum of the lung. The pulmonary artery, pulmonary vein and bronchus were separated carefully using hemostatic forceps and peanut sponges. The method and order of clipping and cutting off of vessels was the same as for the VATS group. Warm physiological saline was used to check the bronchus ligation and syringe the thoracic cavity; this solution was then extracted using a vacuum absorber.

Monitoring and postoperative care. Postoperative care consisted of cefazolin (20 mg/kg, IM) twice daily for 7 days and topical erythromycin ointment applied to the incision sites twice daily for 7 days. Tramadol $(2 \mathrm{mg} / \mathrm{kg}$ ) was administrated intravenously following surgery and once a day for 3 days.

Water was offered when the animal was ambulatory and moistened dog food was offered $6 \mathrm{~h}$ following surgery. 
The following parameters were recorded: HR, RF, rectal temperature, BP (preoperative, every 15 min intraoperatively, postoperatively and on postoperative days 1, 2, 3, 5 and 7), operating time, incision size, postoperative complications and time of standing up. Hematology examination including white blood cells (WBC), red blood cells (RBC), lymphocytes (LY) and granulocytes (Gran) was conducted preoperatively, postoperatively and on days 1, 2, 3, 5, 7 and 14 following surgery.

Blood samples were collected (preoperatively, postoperatively, $4 \mathrm{~h}$, and 1, 3, 5, 7 and 14 days following surgery) through the precaval vein and were centrifuged for $15 \mathrm{~min}$ at $1,000 \mathrm{x} g$ at room temperature $\left(20^{\circ} \mathrm{C}\right)$. Serum for APP analysis was stored at $-80^{\circ} \mathrm{C}$ and the concentration of APPs was assayed using commercially available ELISA kits (BD Pharmingen, San Diego, CA, USA) with specific monoclonal antibodies according to the manufacturer's protocol. Antibodies against the following were used: CRP (51-934DCRP1; 1:100; BD Pharmingen), SAA (F02520; 1:100; Shanghai Westang Bio-Tech Co., Ltd., Shanghai, China) and YKL-40 (F03132; 1:100; Shanghai Westang Bio-Tech Co., Ltd.). Levels of CRP, SAA and YKL-40 were measured preoperatively and postoperatively on days $1,2,3,5,7$ and 14 .

Statistical analysis. Standard statistical methods were used to analyze data. Data are presented as mean \pm standard deviation. Statistical differences within each group were determined by two-way analysis of variance. The paired-sample t-test was used to compare the two groups. $\mathrm{P}<0.05$ was determined to represent statistically significant differences. Statistical analyses were performed using SPSS software (version 22; IBM SPSS, Armonk, NY, USA).

\section{Results}

Animals. All animals survived the follow-up period of 14 days. Total pneumonectomy via VATS and open surgery was successfully performed, with complete removal of all left lobes in 14 animals. No difference was observed in the body weight of dogs in either group (VATS $21.7 \pm 10.5 \mathrm{~kg}$ vs. open $20.4 \pm 3.8 \mathrm{~kg} ; \mathrm{P}>0.05)$. The operating time for the VATS procedure was significantly longer than for the open procedure (VATS 176.7 \pm 22.7 min vs. open $132.4 \pm 15.7 \mathrm{~min}, \mathrm{P}<0.05$ ).

None of the animals in either group had intra- or peri-operative complications. There was no evidence of hemorrhage and no areas of iatrogenic trauma from introduction of surgical instruments. The total length of all skin incisions was $2.5-4 \mathrm{~cm}$ in the VATS group and $5.5-8 \mathrm{~cm}$ in the open group. No other abnormalities were identified in either group. All dogs were considered to have returned to their normal activity levels $36 \mathrm{~h}$ following surgery.

Physiological parameters. Rectal temperature was measured prior to surgery (baseline) and at the designated intra- and post-operative time points (Fig. 1A). Rectal temperature in the VATS group 105, 120 and 135 min postoperation was significantly lower than baseline $(\mathrm{P}<0.05)$. There was no significant change in rectal temperature at any time point in the open group. Three dogs in the VATS and four dogs in the open group experienced hyperpyrexia day 1 and 2 postoperation, but returned to baseline 5 days following surgery.
Comprehensive hemodynamic changes were recorded in all 14 animals. There were no significant changes in HR at any time point in either group (Fig. 1B). Respiratory frequency at 1 day was significantly higher in the open group than the baseline $(\mathrm{P}<0.05)$, and no significant changes were observed among groups (Fig. 1C).

No significant change was observed in systolic BP at any time point in either group (Fig. 2A). During the operative period, the diastolic BP and mean arterial pressure demonstrated a declining trend in both groups, and increased gradually back to baseline levels postoperatively (Fig. 2B and C, respectively). Diastolic BP at 120 min postoperation was significantly decreased in the VATS group compared with baseline (Fig. 2B; $\mathrm{P}<0.05)$.

Hematology. The WBC count in both groups was significantly increased 1, 2 and 3 days following surgery $(\mathrm{P}<0.05)$, and returned to a normal level 2 weeks later (Fig. 3A). Furthermore, it was significantly higher 1 day after surgery in the open group, compared with the VATS group $(\mathrm{P}<0.05)$. There was no significant change in the level of RBCs prior to or following the procedure in both groups (Fig. 3B). The LY count demonstrated an increased trend postoperatively (Fig. 3C; P>0.05) and the Gran count was significantly increased 1 day following surgery (Fig. 3D; $\mathrm{P}<0.05$ ).

Acute-phase proteins. Serum concentrations of CRP and SAA in both groups were significantly increased 1 day after surgery compared with baseline $(\mathrm{P}<0.05$; Fig. $4 \mathrm{~A}$ and $\mathrm{B})$. These decreased gradually back to baseline by day 14 post-surgery (Fig. 4A and B). The serum concentration of CRP was significantly higher on day 3 following surgery in the open group, compared with the VATS group $(\mathrm{P}<0.05$; Fig. 4A). In both groups, the serum concentration of YKL-40 was not significantly decreased at $4 \mathrm{~h}$ following surgery but significantly increased day 1 postoperation compared with baseline $(\mathrm{P}<0.05$; Fig. $4 \mathrm{C})$ and returned to normal level day 5 postoperation. No significant difference in SAA and YKL-40 was observed between the two groups at any time point (Fig. 4B and C).

Arterial blood gas. Arterial blood gas analysis was conducted in the animals (Fig. 5). There was no significant change in the partial pressure of oxygen $\left(\mathrm{PaO}_{2}\right)$ before and after the procedure in either group (Fig. 5B). The pH in both groups decreased immediately following the procedure, and returned to preoperative levels day 1 postoperation $(\mathrm{P}>0.05$; Fig. $5 \mathrm{C})$. The partial pressure of carbon dioxide $\left(\mathrm{PaCO}_{2}\right)$ was elevated immediately following the procedure in both groups; $\mathrm{PaCO}_{2}$ returned to preoperative level day 1 postoperation in the VATS group and day 3 postoperation in the open group ( $P>0.05$; Fig. $5 \mathrm{~A})$. There was no significant difference between the two groups in the values of $\mathrm{pH}, \mathrm{PaO}_{2}$, and $\mathrm{PaCO}_{2}$ at any time point (Fig. 5).

\section{Discussion}

Lung lobes may be excised through either open thoracotomy or VATS. In the present study, pneumonectomy in the hemithorax was performed successfully using both of these approaches without major intra- or post-operative complications. Thus, 
A

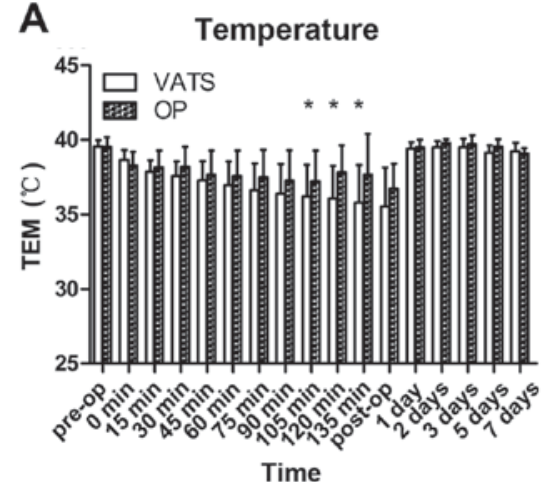

B
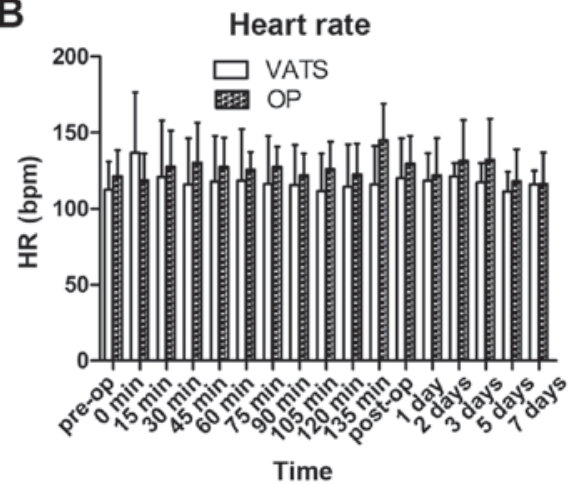

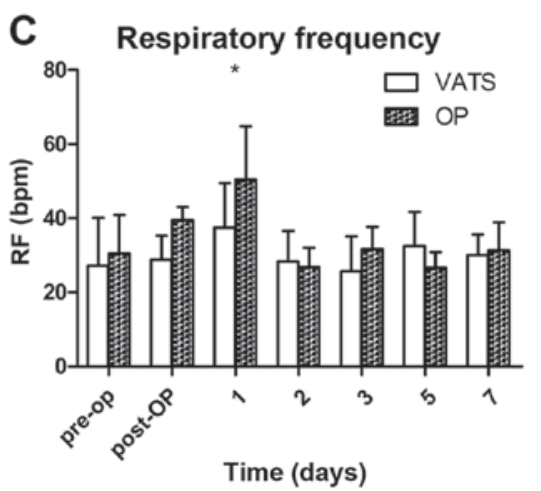

Figure 1. Physiological parameters assessed in all animals. Changes in (A) temperature, (B) HR and (C) RF in 14 dogs that underwent pneumonectomy ( $\mathrm{n}=7$ for VATS and $\mathrm{n}=7$ for open thoracotomy). ${ }^{*} \mathrm{P}<0.05$ vs. preoperative value (baseline). HR, heart rate; TEM, temperature; RF, respiratory frequency; OP, open surgery; VATS, video-assisted thoracoscopic surgery; bpm, beats per minute; pre-op, preoperation; post-op, postoperation.

both VATS and the transthoracic approach are viable alternative techniques to total pneumonectomy. The current study also performed thoracic exploration, surgical lung biopsy and pericardial window creation successfully with the VATS approach in dogs.

Pneumonectomy is a widely performed surgical technology that is used to cure pathological conditions including lung tumors, congenital lung anomalies, chronic lung collapse, chronic progressive lung inflammation, post-traumatic diffuse parenchymal laceration and bronchial rupture. VATS is considered to be the most significant progress made in thoracic surgery at the end of the 20th century. Since the first pioneering lobectomy completed using VATS $(14,15)$, the techniques and reliability of such surgeries have considerably improved (16-19). VATS is now well established as an alternative treatment to open thoracotomy for major resections of lung cancer and benign disease. VATS has the advantage of providing an improved visual field and cosmesis. Previous studies have verified that VATS has other advantages, such as a lower concentration of inflammatory cytokines (20), a lower risk of developing chest infection, reduced pain and improved lung function $(21,22)$. An increasing body of evidence has indicated that patients undergoing VATS experience a shorter hospital stay, have their chest tubes removed earlier $(23,24)$ and have an improved prognosis (25). Aujesky et al (26) observed good outcomes in patients who underwent video-assisted laparoscopic resection of the esophagus for carcinoma following neoadjuvant therapy.

Body temperature decreased during all surgeries in the current study. A decrease in rectal temperature was observed postoperatively in both groups; although heating blankets may help, intraoperative hypothermia is inevitable. A lower rectal temperature was observed postoperatively in the VATS group compared with the open group, this is potentially due to the operating time in the VATS group being much longer.

WBC, Gran and LY are factors that influence inflammation and stress. The serum level of these factors was raised on day 1 following surgery; this may be due to the severe trauma caused by surgery and anesthesia. However, the inflammatory reaction reflected through WBC difference on day 1 suggested that the thoracotomy caused more severe body stress, as the longer incision increases the likelihood of infection. Considering this with the change in APPs, no influence on the dogs owing to a longer operating time or a bigger incision was observed; however, suturing the incision took longer in the open surgery.

APPs may cause rapid response to infections, burns, invasive surgery, inflammation and tissue injury stress. Levels of the APPs CRP, SAA, and YKL-40 increase as a result of the inflammatory response to infection or tissue damage, and have been used to evaluate surgery technique, infection and pathology progress. CRP and SAA levels increase in a number of pathological states, including surgical trauma (27-30), esophageal neoplasia, infection with H3N2 swine influenza virus, alcoholic liver and systemic inflammation (11,31-34). In the present study, levels of all APPs increased significantly on day 1 postoperatively compared with baseline (preoperative levels) and decreased over the subsequent time point measurements. This change suggested that the surgery resulted in tissue trauma and inflammation. Although animals in the thoracotomy group exhibited more injury and pain postoperatively, VATS required more surgical manipulation and therefore, resulted in a longer time under anesthesia. This may explain the result observed in 
A

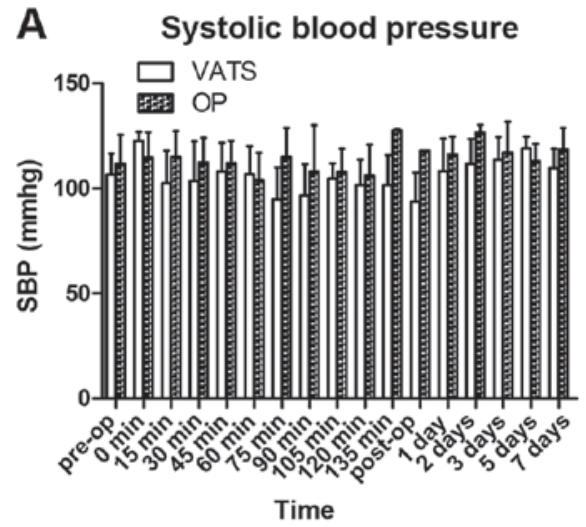

B Diastolic blood pressure

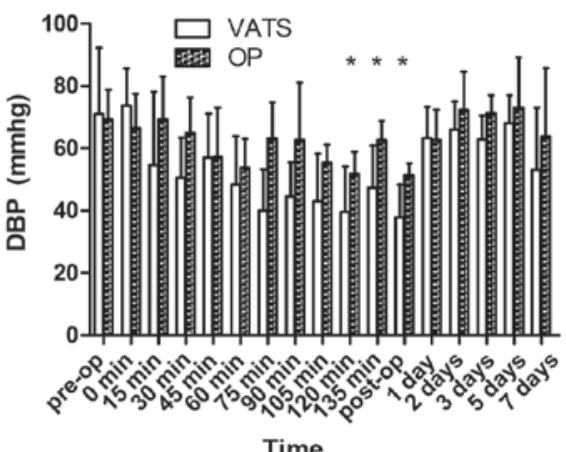

Time

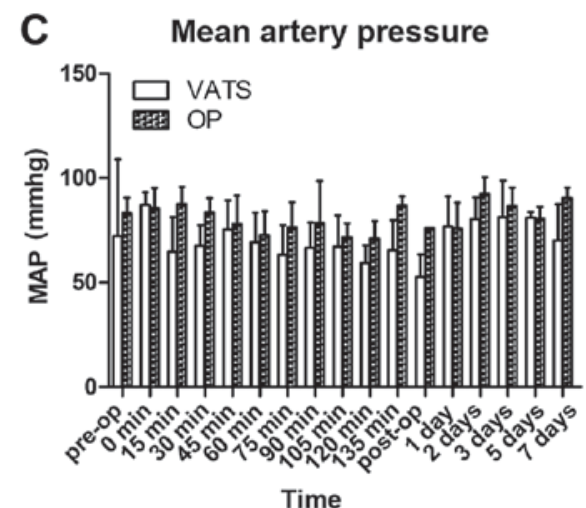

Figure 2. Hemodynamic changes assessed in all animals. Changes in (A) SBP, (B), DBP and (C) MAP in 14 dogs that underwent pneumonectomy (n=7 for VATS and $n=7$ for open thoracotomy). ${ }^{*}<0.05$ vs. preoperative value (baseline). SBP, systolic blood pressure; DBS, diastolic blood pressure; MAP, mean artery pressure; OP, open surgery; VATS, video-assisted thoracoscopic surgery; pre-op, preoperation; post-op, postoperation.
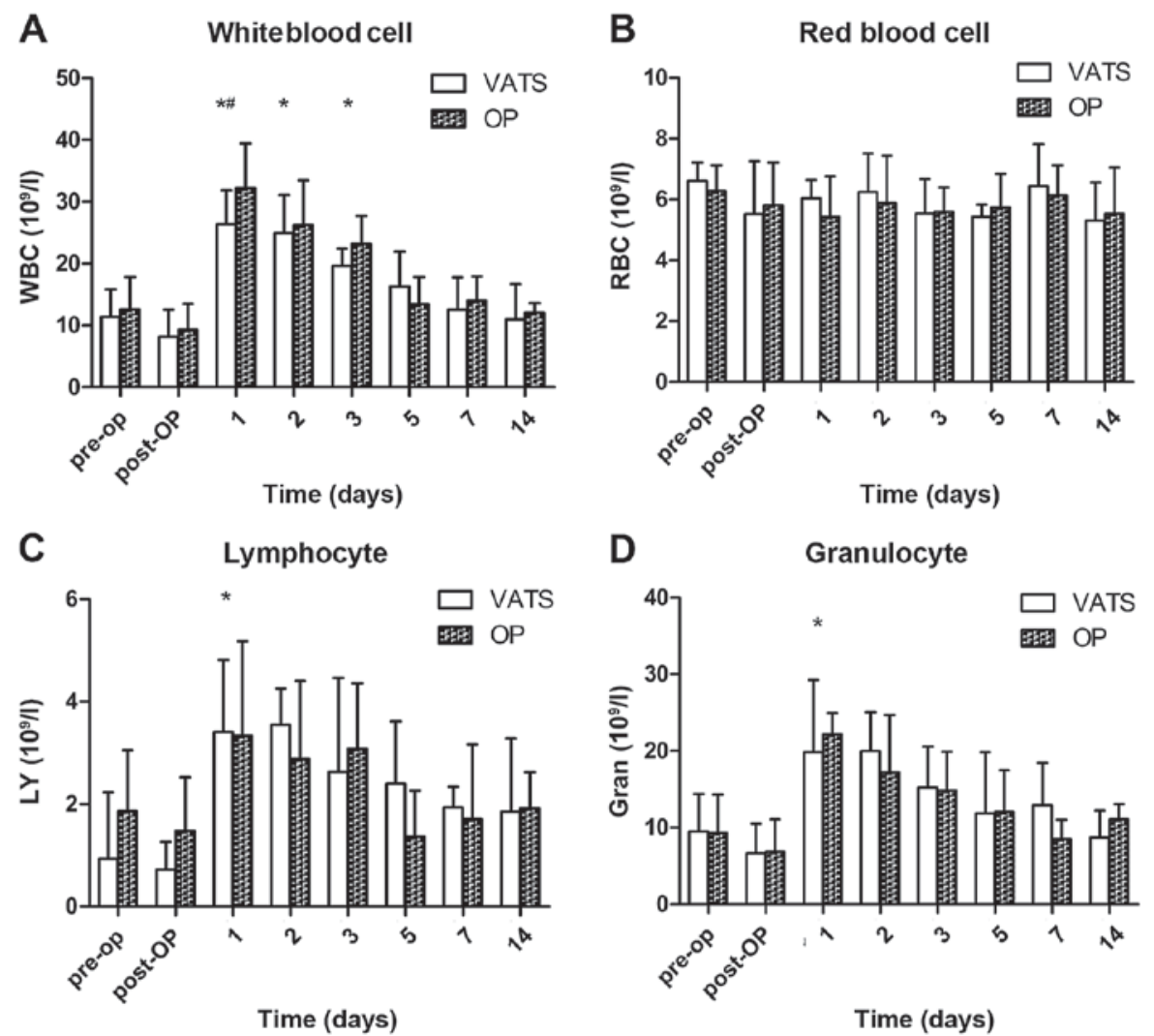

Figure 3. Hematology assessments in all animals. Changes in (A) WBCs, (B) RBCs, (C) LYs and (D) Grans in 14 dogs that underwent pneumonectomy (n=7 for VATS and $\mathrm{n}=7$ for open thoracotomy). ${ }^{*} \mathrm{P}<0.05$ vs. preoperative value (baseline) and ${ }^{\text {"}} \mathrm{P}<0.05$ for VATS vs. transthoracic approach at the same time point. WBC, white blood cells; RBD, red blood cells; LY, lymphocytes; Gran, granulocyes; OP, open surgery; VATS, video-assisted thoracoscopic surgery; pre-op, preoperation; post-op, postoperation. 

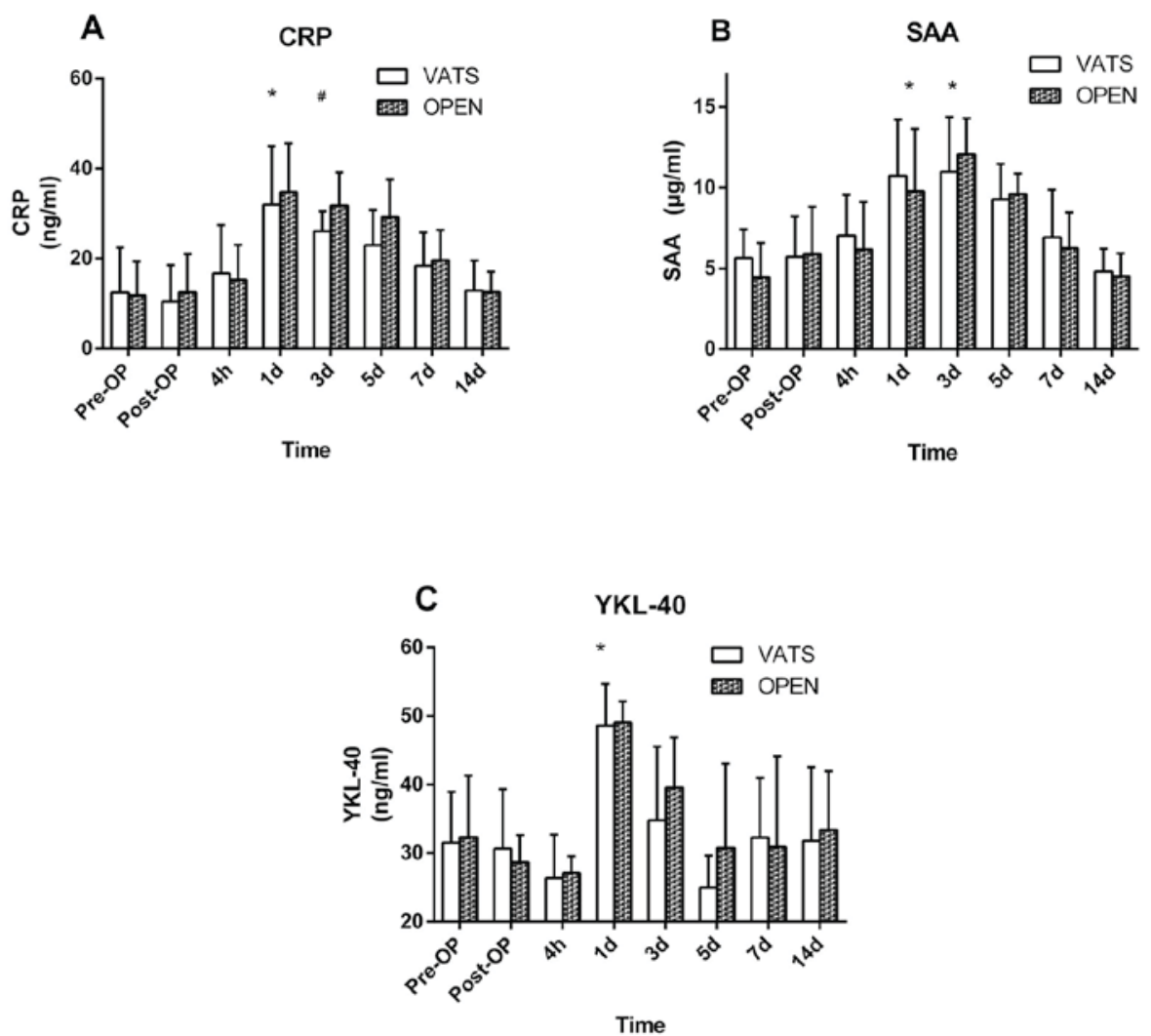

Figure 4. Assessment of serum concentrations in all animals. Changes in (A) CRP, (B) SAA and (C) YKL-40 in 14 dogs that underwent pneumonectomy (n=7 for VATS and $\mathrm{n}=7$ for open thoracotomy). ${ }^{*} \mathrm{P}<0.05$ vs. preoperative value (baseline) and ${ }^{\#} \mathrm{P}<0.05$ for VATS vs. transthoracic approach at the same time point. CRP, C reactive protein; SAA, serum acute-phase protein; OP, open surgery; VATS, video-assisted thoracoscopic surgery; pre-op, preoperation; post-op, postoperation.

A

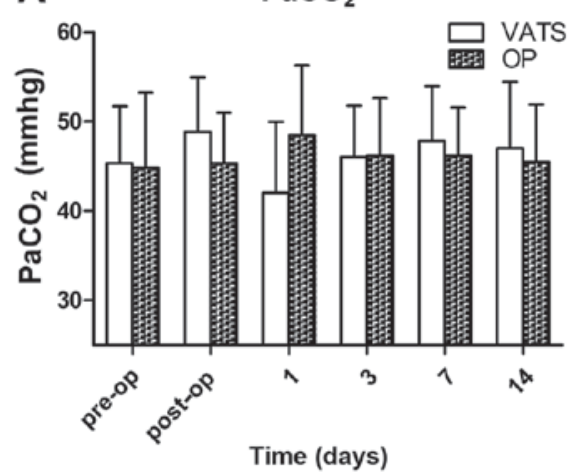

C
B

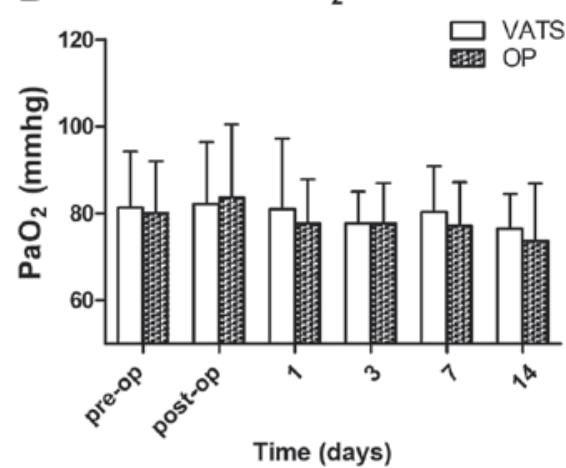

$\mathrm{pH}$

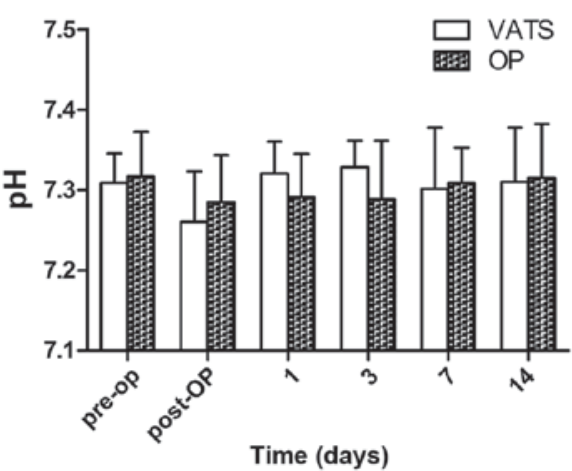

Figure 5. Arterial blood gas analysis in all animals. Changes in (A) $\mathrm{PaCO}_{2}$, (B) $\mathrm{PaO}_{2}$ and $(\mathrm{C}) \mathrm{pH}$ in 14 dogs that underwent pneumonectomy (n=7 for VATS and $\mathrm{n}=7$ for open thoracotomy). $\mathrm{PaCO}_{2}$, partial pressure of carbon dioxide; $\mathrm{PaO}_{2}$, partial pressure of oxygen; OP, open surgery; VATS, video-assisted thoracoscopic surgery; pre-op, pre-operation; post-op, postoperation. 
the present study; that the serum concentration of CRP in the VATS group was significantly higher compared with the open group on day 3 postoperation, and that no differences were observed between the two groups at other time points.

YKL-40 is not only an APP, but also a biomarker closely associated with respiratory function. Elevated plasma YKL-40 is a prognostic indicator in patients with idiopathic pulmonary arterial hypertension (35), predicts bronchiolitis obliterans development in lung transplant recipients (36), predicts poor prognosis in hepatocellular carcinoma (37) and is elevated in patients with chronic obstructive pulmonary disease and activated alveolar macrophages (38). Therefore, although YKL-40 is not as sensitive as CRP and SAA in detecting surgical trauma and inflammation, the changing concentration of YKL-40 reflects the condition of the respiratory system. In the present study, the change in YKL-40 was similar to the changes in CRP and SAA as a result of body stress and inflammation; no difference was observed between the two groups, indicating that VATS did not have a greater effect on the respiratory system than open thoracotomy.

Thoracic cavity surgery causes pulmonary tissue damage and a decrease in respiratory function, which is reflected by the change in blood gases. There was a slight change in blood gases in all animals in the current study as a result of mechanical ventilation. Significant changes in blood gases during laparoscopic surgery have been reported owing to pneumoperitoneum; therefore, use of the endoscope in the thoracic cavity in the current study did not cause the complication of pneumoperitoneum.

Standard pleural drainage was not used in the present study. Leakage or pneumothorax was not observed in any dogs. Chest drain use remains controversial, as thoracotomy incisions may damage the intercostal nerves and lead to chronic neuropathy (39). Satherley et al (40) reported that the use of an intercostal chest drain following lung biopsy increased the period of hospitalization and Nakashima et al (41) reported that postoperative morbidity did not increase following thoracoscopic lung wedge resection without a chest tube. Luckraz et al (42) demonstrated that there was no requirement for an intercostal chest drain in patients that had received VATS lung resection if no air leakage was noted during surgery. Intraoperative blood loss was not recorded in the current study, as there was very minimal intraoperative bleeding in both groups; however, the blood loss observed in the VATS group was subjectively less than in the open group. The reason for this may be the improved visual field and accuracy of surgery due to the magnified image provided by the endoscope in VATS.

A number of previous studies have demonstrated the feasibility of the natural orifice transluminal endoscopic surgery (NOTES) approach (transgastric, transumbilical, transoral, transvaginal) applied in thoracic surgery and indicate that NOTES caused less stress than the open approach (43-46). The NOTES approach reportedly resulted in improved cosmetic outcomes compared with VATS, laparoscopic surgery and open surgery (47). However, a number of NOTES approaches in the abdominal cavity (including gastric, uterine, and umbilical) damaged the diaphragm and the surgical path was affected by the liver (48-51). Further investigations are required to compare the difference between NOTES and VATS in thoracic surgery.
In conclusion, no significant difference between VATS and open pneumonectomy was observed in dogs following an analysis in the change to APPs and hemodynamics. Animals in the VATS group experienced a longer surgical time and a smaller incision scar. No complication was observed in either group. Therefore, VATS is a feasible and safe approach for pneumonectomy in dogs.

\section{Acknowledgements}

The present study was supported by the National Natural Science Foundation of China (grant nos. 31272617 and 31472245).

\section{References}

1. Liu L, Zhang LJ, Chen B, Cao JM, Lu GM, Yuan L, Li K and $\mathrm{Xu}$ J: Novel CT-guided coil localization of peripheral pulmonary nodules prior to video-assisted thoracoscopic surgery: A pilot study. Acta Radiol 55: 699-706, 2014.

2. Panagopoulos N, Papavasileiou G, Koletsis E, Kastanaki M and Anastasiou N: VATS bullectomy and apical pleurectomy for spontaneous pneumothorax in a young patient with Swyer-James-Mc Leod syndrome: Case report presentation and literature review focusing on surgically treated cases. J Cardiothorac Surg 9: 13, 2014.

3. Liu CY, Chu Y, Wu YC, Yuan HC, Ko PJ, Liu YH and Liu HP: Transoral endoscopic surgery versus conventional thoracoscopic surgery for thoracic intervention: Safety and efficacy in a canine survival model. Surg Endosc 27: 2428-2435, 2013.

4. Jackson J, Richter KP and Launer DP: Thoracoscopic partial pericardiectomy in 13 dogs. J Vet Intern Med 13: 529-533, 1999.

5. Fujimoto T, Zaboura G, Fechner S, Hillejan L, Schröder T, Marra A, Krbek T, Hinterthaner M, Greschuchna D and Stamatis G: Completion pneumonectomy: Current indications, complications and results. J Thorac Cardiovasc Surg 121: 484-490, 2001

6. Mcgovern EM, Trastek VF, Pairolero PC and Payne WS: Completion pneumonectomy: Indications, complications, and results. Ann Thoracic Surg 46: 141-146, 1988.

7. Gonzalez-Zamora JF, Perez-Guille B, Soriano-Rosales RE, Jimenez-Bravo-Luna MA, Gutierrez-Castrellon P, Ridaura-Sanz C and Alvarez FV: Video-assisted thoracoscopy for diaphragmatic plication: Experimental study in a canine model. J Laparoendosc Adv Surg Tech A 15: 661-666, 2005.

8. Case JB, Mayhew PD and Singh A: Evaluation of video-assisted thoracic surgery for treatment of spontaneous pneumothorax and pulmonary bullae in dogs. Vet Surg 44 (Suppl 1): S31-S38, 2015.

9. Bodner J: Video-assisted thoracoscopic (VATS) sublobar anatomic resections for lung cancer. Zentralbl Chirur 139: 102-107, 2014 (In German).

10. Lyscov A, Obukhova T, Ryabova V, Sekhniaidze D, Zuiev V and Gonzalez-Rivas D: Double-sleeve and carinal resections using the uniportal VATS technique: A single centre experience. J Thorac Dis 8 (Suppl 3): S235-S241, 2016.

11. Nivy R, Caldin M, Lavy E, Shaabon K, Segev G and Aroch I: Serum acute phase protein concentrations in dogs with spirocercosis and their association with esophageal neoplasia-a prospective cohort study. Vet Parasitol 203: 153-159, 2014.

12. The Animal Welfare Acts: US PL 89-544; 91-579; 94-279; and PL 99-198.

13. American Veterinary Medical Association Guidelines for the Euthanasia of Animals: 2013 Edition. American Veterinary Medical Association, Schaumburg, IL, USA

14. Lewis RJ, Sisler GE and Caccavale RJ: Imaged thoracic lobectomy: Should it be done? Ann Thorac Surg 54: 80-83, 1992.

15. Kirby TJ, Mack MJ, Landreneau RJ and Rice TW: Initial experience with video-assisted thoracoscopic lobectomy. Ann Thorac Surg 56: 1248-1253, 1993.

16. Tsang FH, Chung SS and Sihoe AD: Video-assisted thoracic surgery for bronchopulmonary sequestration. Interact Cardiovasc Thorac Surg 5: 424-426, 2006.

17. Shiraishi T, Shirakusa T, Miyoshi T, Hiratsuka M, Yamamoto $S$ and Iwasaki A: A completely thoracoscopic lobectomy/segmentectomy for primary lung cancer-technique, feasibility, and advantages. Thorac Cardiovasc Surg 54: 202-207, 2006. 
18. Oda M, Ishikawa N, Tsunezuka Y, Matsumoto I, Tamura M, Kawakami K and Watanabe G: Closed three-port anatomic lobectomy with systematic nodal dissection for lung cancer. Surg Endosc 21: 1464-1465, 2007.

19. Tajiri M, Maehara T, Nakayama H and Sakamoto K: Decreased invasiveness via two methods of thoracoscopic lobectomy for lung cancer, compared with open thoracotomy. Respirology 12: 207-211, 2007.

20. Yim AP, Wan S, Lee TW and Arifi AA: VATS lobectomy reduces cytokine responses compared with conventional surgery. Ann Thorac Surg 70: 243-247, 2000.

21. Phan K and Yan TD: VATS segmentectomy for pulmonary metastasis. Ann Cardiothorac Surg 3: 192-193, 2014.

22. Cao C, Manganas C, Ang SC, Peeceeyen S and Yan TD: Video-assisted thoracic surgery versus open thoracotomy for non-small cell lung cancer: A meta-analysis of propensity score-matched patients. Interact Cardiovasc Thorac Surg 16 244-249, 2013.

23. Scott WJ, Matteotti RS, Egleston BL, Oseni S and Flaherty JF: A comparison of perioperative outcomes of video-assisted thoracic surgical (VATS) lobectomy with open thoracotomy and lobectomy: Results of an analysis using propensity score based weighting. Ann Surg Innov Res 4: 1, 2010.

24. Yang X, Wang S and Qu J: Video-assisted thoracic surgery (VATS) compares favorably with thoracotomy for the treatment of lung cancer: A five-year outcome comparison. World J Surg 33: $1857-1861,2009$.

25. Sawada S, Komori E, Yamashita M, Nakata M, Nishimura R, Teramoto N, Segawa Y and Shinkai T: Comparison in prognosis after VATS lobectomy and open lobectomy for stage I lung cancer: Retrospective analysis focused on a histological subgroup. Surg Endosc 21: 1607-1611, 2007.

26. Aujesky R,NeoralC,Kral V,Bohanes T, Vrba R and Vomackova K: Video-assisted laparoscopic resection of the esophagus for carcinoma after neoadjuvant therapy. Hepatogastroenterology 56 : $1035-1038,2009$

27. Chung YG, Won YS, Kwon YJ, Shin HC, Choi CS and Yeom JS Comparison of serum CRP and procalcitonin in patients after spine surgery. J Korean Neurosurg Soc 49: 43-48, 2011.

28. Planellas M, Cuenca R, Tabar MD, Bertolani C, Poncet C, Closa JM, Lorente J, Cerón JJ and Pastor J: Clinical assessment and C-reactive protein (CRP), haptoglobin (Hp) and cardiac troponin I (cTnI) values of brachycephalic dogs with upper airway obstruction before and after surgery. Can J Vet Res 79: $58-63,2015$.

29. Syeda T, Hashim AS, Rizvi HA and Hadi SM: Pre- and post-operative values of serum CRP in patients undergoing surgery for brain tumour. J Pak Med Assoc 64: 271-274, 2014.

30. Neumaier M, Metak G and Scherer MA: C-reactive protein as a parameter of surgical trauma: CRP response after different types of surgery in 349 hip fractures. Acta Orthop 77: 788-790, 2006.

31. Kim SR, Kondo F, Otono Y, Imoto S, Ando K, Hirakawa M Fukuda K, Sasaki M, Kim SK, Komaki T, et al: Serum amyloid A and C-reactive protein positive nodule in alcoholic liver cirrhosis, hard to make definite diagnosis. Hepatol Res 44: 584-590, 2014.

32. Pomorska-Mól M, Kwit K, Pejsak Z and Markowska-Daniel I Analysis of the acute-phase protein response in pigs to clinical and subclinical infection with $\mathrm{H} 3 \mathrm{~N} 2$ swine influenza virus. Influenza Other Respir Viruses 8: 228-234, 2014

33. Christensen MB, Langhorn R, Goddard A, Andreasen EB Moldal E, Tvarijonaviciute A, Kirpensteijn J, Jakobsen S, Persson F and Kjelgaard-Hansen M: Comparison of serum amyloid $\mathrm{A}$ and $\mathrm{C}$-reactive protein as diagnostic markers of systemic inflammation in dogs. Can Vet J 55: 161-168, 2014.

34. Jitpean S, Holst BS, Höglund OV, Pettersson A, Olsson U, Strage E, Södersten F and Hagman R: Serum insulin-like growth factor-I, iron, C-reactive protein, serum amyloid a for prediction of outcome in dogs with pyometra. Theriogenology 82: 43-48, 2014

35. Chen G, Yang T, Gu Q, Ni XH, Zhao ZH, Ye J, Meng XM, Liu ZH, He JG and Xiong CM: Elevated plasma YKL-40 as a prognostic indicator in patients with idiopathic pulmonary arterial hypertension. Respirology 19: 608-615, 2014.
36. Jaksch P, Taghavi S, Klepetko W and Salama M: Pretransplant serum human chitinase-like glycoprotein YKL-40 concentrations independently predict bronchiolitis obliterans development in lung transplant recipients. J Thorac Cardiovasc Surg 148: 273-281, 2014.

37. Zhu CB, Chen LL, Tian JJ, Su L, Wang C, Gai ZT, Du WJ and Ma GL: Elevated serum YKL-40 level predicts poor prognosis in hepatocellular carcinoma after surgery. Ann Surg Oncol 19: 817-825, 2012

38. Létuvé S, Kozhich A, Arouche N, Grandsaigne M, Reed J, Dombret MC, Kiener PA, Aubier M, Coyle AJ and Pretolani M: YKL-40 is elevated in patients with chronic obstructive pulmonary disease and activates alveolar macrophages. J Immunol 181: 5167-5173, 2008

39. Lin TY, Chu Y, Wu YC, Liu CY, Yeh CJ, Hsieh MJ, Yuan HC, Ko PJ, Liu YH and Liu HP: Feasibility of transumbilical lung wedge resection in a canine model. J Laparoendosc Adv Surg Tech A 23: 684-692, 2013.

40. Satherley LK, Luckraz H, Rammohan KS, Phillips M, Kulatilake NE and O'Keefe PA: Routine placement of an intercostal chest drain during video-assisted thoracoscopic surgical lung biopsy unnecessarily prolongs in-hospital length of stay in selected patients. Eur J Cardiothorac Surg 36: 737-740, 2009.

41. Nakashima S, Watanabe A, Mishina T, Obama T, Mawatari T and Higami T: Feasibility and safety of postoperative management without chest tube placement after thoracoscopic wedge resection of the lung. Surg Today 41: 774-779, 2011.

42. Luckraz H, Rammohan KS, Phillips M, Abel R, Karthikeyan S, Kulatilake NE and O'Keefe PA: Is an intercostal chest drain necessary after video-assisted thoracoscopic (VATS) lung biopsy? Ann Thorac Surg 84: 237-239, 2007.

43. Boylu U, Oommen M, Joshi V, Thomas R and Lee BR: Natural orifice translumenal endoscopic surgery (NOTES) partial nephrectomy in a porcine model. Surg Endosc 24: 485-489, 2010.

44. Zhu LH, Chen L, Yang S, Liu D, Zhang J, Cheng X and Chen W: Embryonic NOTES thoracic sympathectomy for palmar hyperhidrosis: Results of a novel technique and comparison with the conventional VATS procedure. Surg Endosc 27: 4124-4129, 2013.

45. Nemani A, Sankaranarayanan G, Olasky JS, Adra S, Roberts KE, Panait L, Schwaitzberg SD, Jones DB and De S: A comparison of NOTES transvaginal and laparoscopic cholecystectomy procedures based upon task analysis. Surg Endosc 28: 2443-2451, 2014.

46. Voermans RP, Sheppard B, van Berge Henegouwen MI, Fockens $\mathrm{P}$ and Faigel DO: Comparison of Transgastric NOTES and laparoscopic peritoneoscopy for detection of peritoneal metastases. Ann Surg 250: 255-259, 2009.

47. Freeman LJ, Rahmani EY, Al-Haddad M, Sherman S, Chiorean MV, Selzer DJ, Snyder PW and Constable PD: Comparison of pain and postoperative stress in dogs undergoing natural orifice transluminal endoscopic surgery, laparoscopic, and open oophorectomy. Gastrointest Endosc 72: 373-380, 2010

48. Wen CT, Chu Y, Yeh CJ, Liu CY, Yuan HC, Ko PJ, Liu YH and Liu HP: Feasibility and safety of endoscopic transumbilical thoracic surgical lung biopsy: A survival study in a canine model. J Surg Res 183: 47-55, 2013 .

49. De Palma GD, Siciliano S, Addeo P, Salvatori F, Persico M, Masone S, Rega M, Maione F, Coppola Bottazzi E, Serrao E, et al: A NOTES approach for thoracic surgery: Transgastric thoracoscopy via a diaphragmatic incision in a survival porcine model. Minerva Chir 65: 11-15, 2010.

50. Chu Y, Liu CY, Wu YC, Hsieh MJ, Chen TP, Chao YK, Wu CY, Yuan HC, Ko PJ, Liu YH and Liu HP: Comparison of hemodynamic and inflammatory changes between transoral and transthoracic thoracoscopic surgery. PLoS One 8: e50338, 2013.

51. Voermans RP, Faigel DO, van Berge Henegouwen MI, Sheppard B and Fockens P: Comparison of transcolonic NOTES and laparoscopic peritoneoscopy for the detection of peritoneal metastases. Endoscopy 42: 904-909, 2010. 